\title{
Chryseobacterium arothri sp. nov., isolated from the kidneys of a pufferfish
}

\author{
Sonia Campbell, Renee M. Harada and Oing X. Li \\ Department of Molecular Biosciences and Bioengineering, University of Hawai'i at Manoa, \\ 1955 East West Road, Honolulu, HI 96822, USA
}

Correspondence

Qing X. Li

qingl@hawaii.edu

\begin{abstract}
A Gram-negative, non-flagellated, rod-shaped bacterium, designated strain $\mathrm{P} 2 \mathrm{~K} 6^{\top}$, was isolated from the kidneys of a pufferfish (Arothron hispidus) caught off the coast of Kaneohe Bay, O'ahu, Hawai'i. The strain formed yellowish colonies when grown on marine agar. Phylogenetic analysis based on $16 \mathrm{~S}$ rRNA gene sequences showed that strain $\mathrm{P} 2 \mathrm{~K} 6^{\top}$ was related most closely to members of the genus Chryseobacterium. Levels of 16S rRNA gene sequence similarity between strain $\mathrm{P} 2 \mathrm{~K}^{\top}{ }^{\top}$ and the type strains of recognized species of the genus Chryseobacterium were $94-96.6 \%$, suggesting that the strain represents a novel species within this genus. The DNA G $+\mathrm{C}$ content of strain $\mathrm{P}_{2} \mathrm{~K}^{\top}{ }^{\top}$ was $36.5 \mathrm{~mol} \%$, the dominant fatty acids were iso- $\mathrm{C}_{15: 0}$ (35.3\%) and iso- $\mathrm{C}_{17: 0} 3-\mathrm{OH}(14.9 \%)$, and the most abundant quinone was menaquinone MK6. On the basis of the data from this polyphasic study, it is suggested that strain $P 2 K 6^{\top}$ represents a novel species of the genus Chryseobacterium, for which the name Chryseobacterium arothri sp. nov. is proposed. The type strain is $\mathrm{P}_{2} \mathrm{~K}^{\top}{ }^{\top}\left(=\mathrm{CIP} 109575^{\top}=\mathrm{DSM} 19326^{\top}\right)$.
\end{abstract}

The genus Chryseobacterium was established based on the reclassification of several species of the genus Flavobacterium (as Chryseobacterium balustinum, C. gleum, C. indologenes, C. indoltheticum, C. meningosepticum and C. scophthalmum; Vandamme et al., 1994). At the time of writing, the genus comprised 16 recognized species, including $C$. hispanicum (Gallego et al., 2006) and C. formosense (Young et al., 2005).

The pufferfish Arothron hispidus is commonly found in the warm tropical waters around the Hawaiian islands. Bacterial strain $\mathrm{P} 2 \mathrm{~K} 6^{\mathrm{T}}$ was isolated from the kidneys of one A. hispidus caught off the windward side of the island of $\mathrm{O}^{\prime}$ ahu in August 2005. Strain $\mathrm{P} 2 \mathrm{~K}^{\mathrm{T}}$ was isolated on half-strength marine agar 2216 (1/2MA; Difco). The yellowish colonies that developed were further purified and maintained in $10 \%$ glycerol $/ 90 \%$ half-strength marine broth at $-80{ }^{\circ} \mathrm{C}$.

A male pufferfish weighing $306 \mathrm{~g}$ was dissected into different organs and body parts. Subsamples of each organ were homogenized with sterile artificial seawater (50$100 \mathrm{ml}$ ) (Coralife) and serial dilutions were plated onto $1 / 2 \mathrm{MA}$. Media were subsequently incubated at $30{ }^{\circ} \mathrm{C}$ for $1-$ 5 days. Genomic DNA was isolated from pure cultures by using an equal volume ratio of phenol/chloroform (Marmur, 1961). Amplification of the 16S rRNA gene from genomic DNA was done via PCR by using primers 27F and 1492R (Lane, 1991) and Mastermix Taq DNA polymerase (Eppendorf). All PCR products

The GenBank/EMBL/DDBJ accession number for the 16S rRNA gene sequence of strain $\mathrm{P} 2 \mathrm{~K}^{\top}{ }^{\top}$ is EF554408. were purified by using the Ultraclean PCR purification kit (Mo Bio Lab) and sequenced by the Advanced Studies of Genomics, Proteomics, and Bioinformatics Center at the University of Hawai' $i$ at Manoa. Sequences were edited manually and assembled by using Seqman II (Lasergene) and subjected to a BLASTN comparison search against sequences in the public domain (Altschul et al., 1997).

The phylogenetic relationship of strain $\mathrm{P} 2 \mathrm{~K}^{\mathrm{T}}{ }^{\mathrm{T}}$ to recognized species of the genus Chryseobacterium was determined based on comparative 16S rRNA gene sequence analyses. These analyses were performed with programs in the PHYLIP 3.63 package (Felsenstein, 2004). Evolutionary distances were calculated by using the maximum-likelihood method with DNADIST. Bootstrap analyses were based on 100 replicates by using the programs SEQBOOT, DNADIST and CONSENSE. Phylogenetic trees were constructed with jumbled orders of the sequences and the neighbourjoining method (Saitou \& Nei, 1987).

Fig. 1 shows the phylogenetic tree based on the 16S rRNA gene. The nearly complete (1399 bp) 16S rRNA gene sequence of strain $\mathrm{P} 2 \mathrm{~K} 6^{\mathrm{T}}$ was related most closely to that of the type strain of $C$. formosense (Young et al., 2005; 96.6\% similarity). Strain $\mathrm{P} 2 \mathrm{~K}^{\mathrm{T}}{ }^{\mathrm{T}}$ showed a level of $16 \mathrm{~S}$ gene sequence similarity to the type strains of C. hispanicum and Chryseobacterium caeni of $96.1 \%$. Based on these low levels of sequence similarity, strain $\mathrm{P} 2 \mathrm{~K}^{\mathrm{T}}$ should be considered as being distinct from recognized species of the genus Chryseobacterium (Stackebrandt \& Goebel, 1994).

Determination of the DNA G $+C$ content was performed by the Deutsche Sammlung von Mikroorganismen und 
Table 1. Fatty acid composition of strain $\mathrm{P} 2 \mathrm{~K} 6^{\top}$ and some recognized species of the genus Chryseobacterium

Strains: 1, P2K6 ${ }^{\mathrm{T}}$ (Chryseobacterium arothri sp. nov.); 2, C. formosense CCUG $49271^{\mathrm{T}}$ (data from Young et al., 2005); 3, C. vrystaatense LMG 22846 ${ }^{\mathrm{T}}$ (De Beer et al., 2005); 4, C. indoltheticum ATCC $27950^{\mathrm{T}}$; 5, C. balustinum LMG $8329^{\mathrm{T}}$; 6, C. gleum NCTC $11432^{\mathrm{T}}$; 7, C. indologenes NCTC $10796^{\mathrm{T}}$; 8 , C. scophthalmum LMG $13028^{\mathrm{T}}$; 9, C. joostei LMG $18212^{\mathrm{T}}$ (data for 4-9 from Hugo et al., 2003); 10, C. defluvii DSM 14219 ${ }^{\mathrm{T}}$ (Kämpfer et al., 2003); 11, C. hispanicum JCM $13554^{\mathrm{T}}$ (Gallego et al., 2006); 12, C. daecheongense DSM 15235 ${ }^{\mathrm{T}}$ (Kim et al., 2005); 13, C. taichungense CCUG 50001 ${ }^{\mathrm{T}}$ (Shen et al., 2005); 14, C. wanjuense DSM $17724^{\mathrm{T}}$ (Weon et al., 2006); 15, C. piscium CCUG $51923^{\mathrm{T}}$ (De Beer et al., 2006); 16, C. soldanellicola NBRC $100864^{\mathrm{T}}$ (Park et al., 2006); 17, C. taeanense NBRC $100863^{\mathrm{T}}$ (Park et al., 2006). Values are percentages of the total fatty acids. - , Not detected; NA, data not available; tr, trace amount $(<1 \%)$.

\begin{tabular}{|c|c|c|c|c|c|c|c|c|c|c|c|c|c|c|c|c|c|}
\hline Fatty acid & 1 & 2 & 3 & 4 & 5 & 6 & 7 & 8 & 9 & 10 & 11 & 12 & 13 & 14 & 15 & 16 & 17 \\
\hline $\mathrm{C}_{14: 0}$ & $\operatorname{tr}$ & - & - & - & - & - & - & - & - & - & - & - & - & - & - & - & - \\
\hline anteiso- $\mathrm{C}_{15: 0}$ & 5.45 & 2.1 & 1.7 & 5.9 & $\operatorname{tr}$ & $\operatorname{tr}$ & $\operatorname{tr}$ & $\operatorname{tr}$ & $\operatorname{tr}$ & 3.2 & 3.6 & 1.0 & 0.7 & $\operatorname{tr}$ & 2.7 & 1.9 & 1.1 \\
\hline $\mathrm{C}_{16: 1} \omega 5 c$ & 2.14 & - & - & - & - & - & - & - & - & - & 5.3 & - & - & - & - & - & - \\
\hline$C_{16: 0}$ & 1.53 & 1.5 & 1.1 & 1.0 & 1.6 & 1.3 & $\operatorname{tr}$ & 1.2 & $\operatorname{tr}$ & 1.3 & 2.4 & 1.8 & 1.3 & - & 1.1 & 1.4 & 1.8 \\
\hline iso- $\mathrm{C}_{15: 0} 2-\mathrm{OH}$ & $\operatorname{tr}$ & $\mathrm{SF} 3^{*}$ & SF 3 & SF 3 & SF 3 & SF 3 & SF 3 & SF 3 & SF 3 & SF 3 & SF 3 & SF 3 & SF 3 & SF 3 & SF 3 & SF 3 & SF 3 \\
\hline $\mathrm{C}_{15: 0} 2-\mathrm{OH}$ & $\operatorname{tr}$ & - & - & - & - & - & - & - & - & - & - & - & - & - & - & - & - \\
\hline iso- $\mathrm{C}_{16: 0} 3-\mathrm{OH}$ & $\operatorname{tr}$ & 1.1 & $\operatorname{tr}$ & 1.3 & $\operatorname{tr}$ & - & - & - & - & $\operatorname{tr}$ & - & $\operatorname{tr}$ & 1.4 & - & 1.3 & $\operatorname{tr}$ & $\operatorname{tr}$ \\
\hline $\mathrm{C}_{16: 0} 3-\mathrm{OH}$ & 5.17 & $\operatorname{tr}$ & 1.3 & $\operatorname{tr}$ & 1.4 & 1.1 & 1.0 & 1.0 & 1.2 & $\operatorname{tr}$ & 4.4 & $\operatorname{tr}$ & 2.6 & $\operatorname{tr}$ & $\operatorname{tr}$ & 1.3 & $\operatorname{tr}$ \\
\hline $\mathrm{C}_{18: 1} \omega 9 c$ & $\operatorname{tr}$ & - & - & - & - & - & - & - & - & - & - & - & - & - & - & - & - \\
\hline iso- $\mathrm{C}_{17: 0}$ & - & 2.3 & $\operatorname{tr}$ & $\operatorname{tr}$ & 1.0 & 1.6 & $\operatorname{tr}$ & $\operatorname{tr}$ & $\operatorname{tr}$ & 2.0 & $\operatorname{tr}$ & 3.0 & $\operatorname{tr}$ & 2.9 & 1.2 & $\operatorname{tr}$ & 1.1 \\
\hline $\mathrm{SF} 4^{\star}$ & 13.89 & - & $\mathrm{NA}$ & NA & $\mathrm{NA}$ & NA & NA & NA & $\mathrm{NA}$ & NA & NA & - & - & - & - & - & - \\
\hline $\mathrm{SF} 5^{\star}$ & 6.23 & - & NA & NA & $\mathrm{NA}$ & NA & NA & NA & $\mathrm{NA}$ & NA & NA & - & - & - & - & - & - \\
\hline SF $6^{*}$ & NA & 6.5 & $\operatorname{tr}$ & 11.2 & 9.2 & 11.8 & 11.1 & 11.5 & 12.1 & 8.4 & $\mathrm{NA}$ & 8.4 & 13.8 & 11.0 & 10.8 & 9.7 & 11.2 \\
\hline
\end{tabular}

${ }^{*}$ Summed feature (SF) 3 contains iso- $\mathrm{C}_{15: 0} 2-\mathrm{OH}$ and/or $\mathrm{C}_{16: 1} \omega 7 c$; summed feature 4 contains $\mathrm{C}_{16: 1} \omega 7 c$ and/or $\mathrm{C}_{16: 1} \omega 6 c$; summed feature 5 contains 10-methyl $\mathrm{C}_{16: 0}$ and/or iso- $\mathrm{C}_{17: 1} \omega 9 c$; and summed feature 6 contains iso- $\mathrm{C}_{15: 0} 2$-OH and/or $\mathrm{C}_{16: 1} \omega 7 c$.

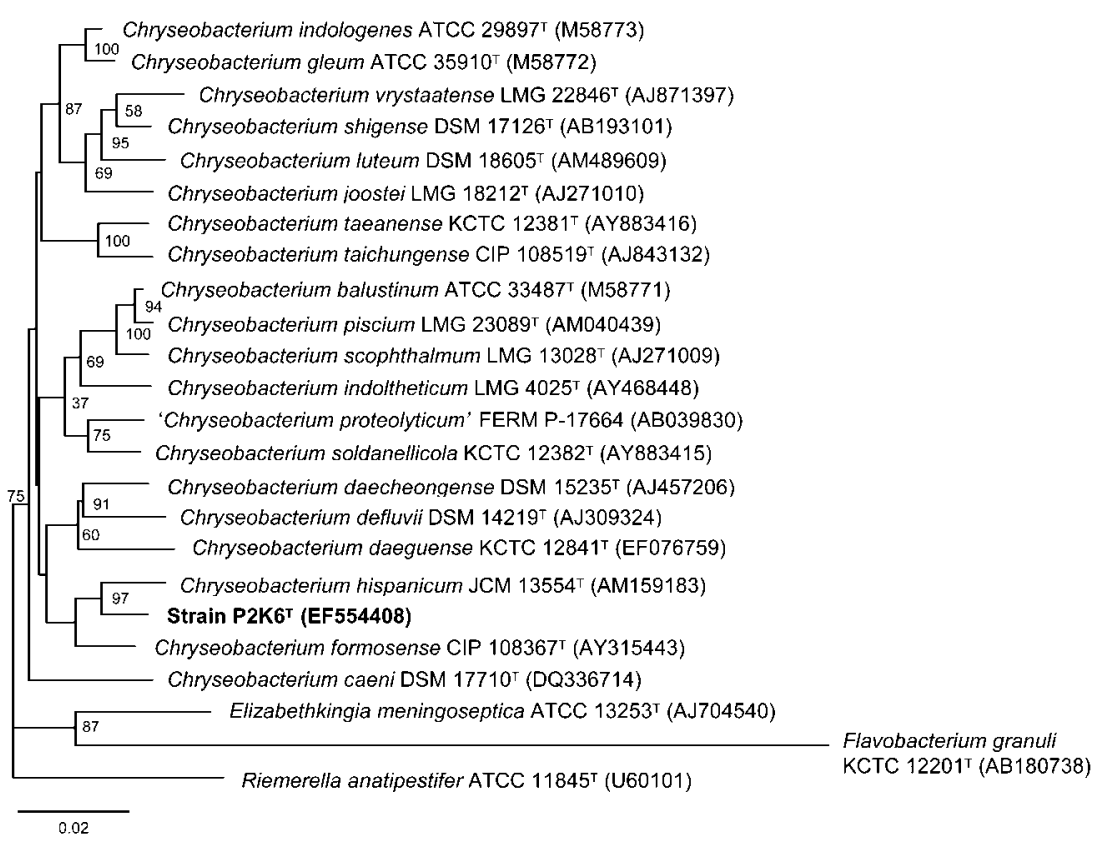

Fig. 1. Neighbour-joining tree based on $16 \mathrm{~S}$ rRNA gene sequences showing the relationship between strain $\mathrm{P} 2 \mathrm{~K}^{\top}$ and some recognized members of the genus Chryseobacterium. Numbers at nodes are bootstrap percentages based on a maximum-likelihood analysis of 100 resampled datasets. The sequences of Elizabethkingia meningoseptica ATCC $13253^{\top}$ and Flavobacterium granuli KCTC $12201^{\top}$ were used as outgroups. Bar, 0.02 nucleotide substitutions per site. 
Table 2. Differential phenotypic characteristics between strain $P 2 K 6^{\top}$ and some recognized species of the genus Chryseobacterium


$50001^{\mathrm{T}}$; 5, C. balustinum LMG $8329^{\mathrm{T}}$; 6, C. daecheongense DSM $15235^{\mathrm{T}} ; 7$, C. defluvii DSM $14219^{\mathrm{T}} ; 8$, C. formosense CCUG $49271^{\mathrm{T}}$; 9 , C. gleum

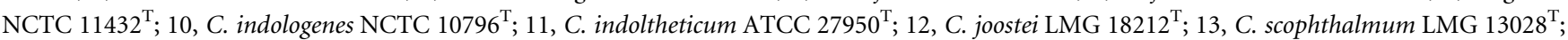
14, C. hispanicum CECT $7129^{\mathrm{T}}$. Data for reference species are from Kämpfer et al. (2003), Hugo et al. (2003), Kim et al. (2005), Shen et al. (2005), Young et al. (2005) and Gallego et al. (2006). +, Positive; -, negative; v, variable; D, delayed; NA, no data.

\begin{tabular}{|c|c|c|c|c|c|c|c|c|c|c|c|c|c|c|}
\hline Characteristic & 1 & 2 & 3 & 4 & 5 & 6 & 7 & 8 & 9 & 10 & 11 & 12 & 13 & 14 \\
\hline \multicolumn{15}{|l|}{ Growth at: } \\
\hline $5{ }^{\circ} \mathrm{C}$ & - & - & - & - & + & - & - & - & + & + & + & + & - & $\mathrm{D}$ \\
\hline $37^{\circ} \mathrm{C}$ & + & + & + & - & $\mathrm{D}$ & - & - & - & - & - & + & + & $\mathrm{D}$ & - \\
\hline $42{ }^{\circ} \mathrm{C}$ & - & - & - & - & - & - & + & - & - & - & - & - & - & - \\
\hline \multicolumn{15}{|l|}{ Enzyme activities } \\
\hline DNase & NA & - & - & NA & + & NA & NA & NA & + & + & + & + & + & - \\
\hline Urease & - & - & - & - & - & - & - & - & - & - & - & $\mathrm{V}$ & + & - \\
\hline Nitrate reduction & - & - & - & - & + & + & - & - & + & - & - & - & - & + \\
\hline Nitrite reduction & - & - & - & NA & - & - & + & NA & + & - & - & - & - & NA \\
\hline Tween 80 hydrolysis & + & - & + & $\mathrm{NA}$ & + & - & NA & - & + & + & + & + & + & - \\
\hline \multicolumn{15}{|l|}{ Acid production from: } \\
\hline L-Arabinose & - & - & - & - & - & - & - & - & + & - & - & - & - & $\mathrm{NA}$ \\
\hline Fructose & + & - & - & $\mathrm{NA}$ & + & + & + & $\mathrm{NA}$ & + & + & - & + & - & + \\
\hline Glycerol & - & - & - & NA & - & + & + & NA & + & + & - & + & - & + \\
\hline Lactose & - & - & - & - & - & - & - & $\mathrm{NA}$ & - & - & - & - & - & - \\
\hline Maltose & + & - & - & + & - & - & + & - & + & + & + & + & - & + \\
\hline Mannitol & - & - & - & - & - & - & - & - & - & - & - & + & - & - \\
\hline Trehalose & - & - & - & + & - & + & + & + & + & + & - & + & + & - \\
\hline DNA G $+C$ content $(\mathrm{mol} \%)$ & 36.5 & 28.8 & 32.1 & NA & 33.1 & 36.6 & 38.8 & NA & 38.0 & 38.5 & 33.8 & 36.8 & 34.2 & 34.3 \\
\hline
\end{tabular}

Zellkulturen (Braunschweig, Germany) by using an HPLC method (Mesbah et al., 1989; Tamaoka \& Komagata, 1984). The DNA G+C content of strain $\mathrm{P} 2 \mathrm{~K}^{\mathrm{T}}{ }^{\mathrm{T}}$ was $36.5 \mathrm{~mol} \%$. Fatty acid methyl ester analysis for strain P2 $6^{\mathrm{T}}{ }^{2}$ was performed by MIDI Laboratories by using whole cells grown on trypticase soy agar (TSA) for $24 \mathrm{~h}$ at $28{ }^{\circ} \mathrm{C}$ (Sasser, 1990). The fatty acid compositions of strain $\mathrm{P} 2 \mathrm{~K}^{\mathrm{T}}$ and of other recognized species of the genus Chryseobacterium are summarized in Table 1 . The major fatty acids for $C$. formosense were iso- $\mathrm{C}_{15: 0}(52.2 \%)$ and iso- $\mathrm{C}_{17: 0} 3-\mathrm{OH}(10.9 \%)$. These were the main two fatty

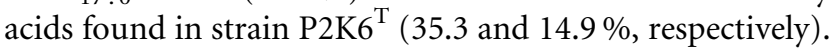
$\mathrm{C}_{16: 1} \omega 5 c$ and $\mathrm{C}_{16: 0} 3-\mathrm{OH}$ were present (2.14 and $5.17 \%$, respectively) in strain $\mathrm{P} 2 \mathrm{~K} 6^{\mathrm{T}}$ but were not detected or were present in trace amounts in $C$. formosense.

Quinone analysis was performed by using fresh whole cells of strain $\mathrm{P} 2 \mathrm{~K}^{\mathrm{T}}{ }^{\mathrm{T}}$ grown in marine broth for 2-3 days. Cells were extracted with a mixture of chloroform/methanol $(2: 1, \mathrm{v} / \mathrm{v})$ (Hiraishi, 1988); after filtration and dilution in acetone, the extract was analysed via HPLC on an Agilent 1100 series chromatograph coupled to a diode array detector and an Agilent model SL single quadrupole mass spectrometer. The HPLC column was a Vydac C18 Mass Spec. Strain P2K6 ${ }^{\mathrm{T}}$ had menaquinone MK6 as the most abundant quinone.

Cells of strain $\mathrm{P} 2 \mathrm{~K}^{\mathrm{T}}{ }^{\mathrm{T}}$ were non-motile, Gram-negative rods. Motility was checked by the hanging drop method by using light microscopy under a $100 \times$ oil-immersion objective, and by stab inoculation into modified motility test agar (per litre distilled water: $10 \mathrm{~g}$ tryptone, $20 \mathrm{~g} \mathrm{NaCl}$ and $5 \mathrm{~g}$ agar). After 2 days growth on $\mathrm{MA}$ at $30^{\circ} \mathrm{C}$, cells were about $0.5 \mu \mathrm{m}$ in diameter. They required salt for growth, which was observed over the range $0.5-3.0 \% \mathrm{NaCl}(\mathrm{w} / \mathrm{v})$, with optimum growth at $2 \% \mathrm{NaCl}$. Growth was observed over a temperature range from $20{ }^{\circ} \mathrm{C}$ (slow growth, 2-3 days) to $37{ }^{\circ} \mathrm{C}$. Growth was tested at temperatures of $5,20,30,37$ and $43{ }^{\circ} \mathrm{C}$ on MA plates over 1 week. Strain $\mathrm{P} 2 \mathrm{~K}^{\mathrm{T}}$ grew anaerobically on TSA (in a Gas-Pak Pouch; Becton Dickinson).

Physiological and biochemical test results are summarized in Table 2. The presence or absence of constitutive enzymes and the substrate profile were determined by using the API ZYM and API 20NE systems (bioMérieux). Oxidation of carbon substrates was determined with Biolog GN microplates (Table 2).

On the basis of 16S rRNA gene sequence data, and distinct morphology, physiology and fatty acid composition, strain $\mathrm{P} 2 \mathrm{~K}^{\mathrm{T}}{ }^{\mathrm{i}}$ is considered to represent a novel species of the genus Chryseobacterium, for which the name Chryseobacterium arothri sp. nov. is proposed.

\section{Description of Chryseobacterium arothri sp. nov.}

Chryseobacterium arothri (a.ro'thri. N.L. n. Arothron scientific genus name of a genus of fish; N. L. gen. n. 
arothri of Arothron, isolated from the pufferfish Arothron hispidus).

Cells are aerobic, facultatively anaerobic, non-motile, Gram-negative rods $(1 \times 0.5 \mu \mathrm{m}$ in size $)$. Circular, yellowish colonies are formed on TSA at $30{ }^{\circ} \mathrm{C}$ after 1 day. Optimum temperature range for growth is $20-37{ }^{\circ} \mathrm{C}$. $\mathrm{NaCl}$ is required for growth, in the range $0.5-3.0 \%(\mathrm{w} / \mathrm{v})$, with optimum growth at $2.0 \% \mathrm{NaCl}$. Utilizes $\alpha$-cyclodextrin, dextrin, glycogen, Tweens 40 and 80, D-cellobiose, Dfructose, gentiobiose, $\alpha$-D-glucose, maltose, D-mannose, Dpsicose, sucrose, turanose, xylitol, pyruvic acid methyl ester, succinic acid monomethyl ester, acetic acid, Dgluconic acid, D-glucosaminic acid, D-glucoronic acid, $\alpha$ hydroxybutyric acid, $\beta$-hydroxybutyric acid, $\alpha$-ketobutyric acid, $\alpha$-ketovaleric acid, propionic acid, quinic acid, D-saccharic acid, L-alaninamide, L-alanine, L-alanyl glycine, L-asparagine, L-glutamic acid, glycyl L-aspartic acid, glycyl L-glutamic acid, hydroxy-L-proline, L-leucine, L-ornithine, L-proline, L-serine, L-threonine, DL-carnitine, $\gamma$-aminobutyric acid, urocanic acid, inosine, uridine, thymidine, $\alpha$-D-glucose 1-phosphate, and D-glucose 6-phosphate as carbon source in Biolog GN2 tests. Produces $\mathrm{H}_{2} \mathrm{~S}$ on triplesugar iron $+2 \% \mathrm{NaCl}$ agar. The predominant fatty acids are iso- $\mathrm{C}_{15: 0}(35.3 \%)$ and iso- $\mathrm{C}_{17: 0} 3-\mathrm{OH}(14.9 \%)$. The DNA G $+\mathrm{C}$ content of the type strain is $36.5 \mathrm{~mol} \%$.

The type strain, $\left.\mathrm{P} 2 \mathrm{~K}^{\mathrm{T}}{ }^{(=\mathrm{CIP}} 109575^{\mathrm{T}}=\mathrm{DSM} 19326^{\mathrm{T}}\right)$, was isolated from the kidneys of a pufferfish Arothron hispidus caught off the coast of the windward side of the island of O'ahu, Hawai'i.

\section{Acknowledgements}

This study was supported in part by a contractual agreement with the State of Hawai'i Department of Health - Office of Hazard Evaluation and Emergency Response. We thank Brian and Myrna Yamane for sample collection.

\section{References}

Altschul, S. F., Madden, T. L., Schäffer, A. A., Zhang, J., Zhang, Z., Miller, W. \& Lipman, D. J. (1997). Gapped BLAST and PSI-BLAST: a new generation of protein database search programs. Nucleic Acids Res 25, 3389-3402.

De Beer, H., Hugo, C. J., Jooste, P. J., Willems, A., Vancanneyt, M., Coenye, T. \& Vandamme, P. (2005). Chryseobacterium vrystaatense sp. nov., isolated from raw chicken in a chicken-processing plant. Int $J$ Syst Evol Microbiol 55, 2149-2153.

De Beer, H., Hugo, C. J., Jooste, P. J., Vancanneyt, M., Coenye, T. \& Vandamme, P. (2006). Chryseobacterium piscium sp. nov., isolated from fish of the South Atlantic Ocean off South Africa. Int J Syst Evol Microbiol 56, 1317-1322.
Felsenstein, J. (2004). PHYLIP (phylogeny inference package), version 3.63. Distributed by the author. Department of Genome Sciences, University of Washington, Seattle, USA.

Gallego, V., Garcia, M. T. \& Ventosa, A. (2006). Chryseobacterium hispanicum sp. nov., isolated from the drinking water distribution system of Sevilla, Spain. Int J Syst Evol Microbiol 56, 1589-1592.

Hiraishi, A. (1988). Respiratory quinone profiles as tools for identifying different bacterial populations in activated sludge. J Gen Appl Microbiol 34, 39-56.

Hugo, C. J., Segers, P., Hoste, B., Vancanneyt, M. \& Kersters, K. (2003). Chryseobacterium joostei sp. nov., isolated from the dairy environment. Int J Syst Evol Microbiol 53, 771-777.

Kämpfer, P., Dreyer, U., Neef, A., Dott, W. \& Busse, H. J. (2003). Chryseobacterium defluvii sp. nov., isolated from wastewater. Int J Syst Evol Microbiol 53, 93-97.

Kim, K. K., Bae, H. S., Schumann, P. \& Lee, S. T. (2005). Chryseobacterium daecheongense sp. nov., isolated from freshwater lake sediment. Int J Syst Evol Microbiol 55, 133-138.

Lane, D. J. (1991). 16S/23S rRNA sequencing. In Nucleic Acid Techniques in Bacterial Systematics, pp. 115-175. Edited by E. Stackebrandt \& M. Goodfellow. Chichester: Wiley.

Marmur, J. (1961). A procedure for the isolation of deoxyribonucleic acid from microorganisms. J Mol Biol 3, 208-218.

Mesbah, M., Premachandran, U. \& Whitman, W. B. (1989). Precise measurement of the $\mathrm{G}+\mathrm{C}$ content of deoxyribonucleic acid by highperformance liquid chromatography. Int J Syst Bacteriol 39, 159-167.

Park, M. S., Jung, S. R., Lee, K. H., Lee, M. S., Do, J. O., Kim, S. B. \& Bae, K. S. (2006). Chryseobacterium soldanellicola sp. nov. and Chryseobacterium taeanense sp. nov., isolated from roots of sand-dune plants. Int J Syst Evol Microbiol 56, 433-438.

Saitou, N. \& Nei, M. (1987). The neighbor-joining method: a new method for reconstructing phylogenetic trees. Mol Biol Evol 4, 406-425.

Sasser, M. (1990). Identification of bacteria by gas chromatography of cellular fatty acids, MIDI Technical Note 101. Newark, DE: MIDI Inc.

Shen, F. T., Kämpfer, P., Young, C. C., Lai, W. A. \& Arun, A. B. (2005). Chryseobacterium taichungense sp. nov., isolated from contaminated soil. Int J Syst Evol Microbiol 55, 1301-1304.

Stackebrandt, E. \& Goebel, B. M. (1994). Taxonomic note: a place for DNA-DNA reassociation and $16 \mathrm{~S}$ rRNA sequence analysis in the present species definition in bacteriology. Int J Syst Bacteriol 44, 846-849.

Tamaoka, J. \& Komagata, K. (1984). Determination of DNA base composition by reversed-phase high-performance liquid chromatography. FEMS Microbiol Lett 25, 125-128.

Vandamme, P., Bernardet, J.-F., Segers, P., Kersters, K. \& Holmes, B. (1994). New perspectives in the classification of the flavobacteria: description of Chryseobacterium gen. nov., Bergeyella gen. nov., and Empedobacter nom. rev. Int J Syst Bacteriol 44, 827-831.

Weon, H. Y., Kim, B. Y., Yoo, S. H., Kwon, S. W., Cho, Y. H., Go, S. J. \& Stackebrandt, E. (2006). Chryseobacterium wanjuense sp. nov., isolated from greenhouse soil in Korea. Int J Syst Evol Microbiol 56, 1501-1504.

Young, C.-C., Kämpfer, P., Shen, F.-T., Lai, W.-A. \& Arun, A. B. (2005). Chryseobacterium formosense sp. nov., isolated from the rhizosphere of Lactuca sativa L. (garden lettuce). Int J Syst Evol Microbiol 55, 423-426. 